\title{
Quantification of Sub-resolution Sized Targets in Cell Fluorescent Imaging
}

\author{
Julien Ghaye, Giovanni De Micheli, Sandro Carrara \\ Integrated Systems Laboratory \\ École Polytechnique Fédérale de Lausanne, EPFL \\ E-mail: julien.ghaye@epfl.ch
}

\begin{abstract}
We introduce two methods for quantifying and evaluating the amount of surface receptors within a group of cells from fluorescence microscope images. First, the average fluorescence intensity method (AFIM), based on the fluorescent pixels average intensity, shows interesting properties for quantifying variations of the amount of surface receptors. It however shows an inherent limit coming form pixels saturation. Second, the amount of fluorescent pixels method (AFPM) is based on the amount of fluorescent pixels by modeling its relation with the amount of surface receptors. The established nonlinear model is a tool for quantitatively evaluating the amount of receptors. The images used for establishing and developing these methods are originating from a simulated environment. Synthetic images featuring simulated cells with fluorescentlystained surface receptors were used. The two methods have been carefully evaluated based on those synthetic images.
\end{abstract}

\section{INTRODUCTION}

Fluorescence microscopy is a basic and useful tool for insitu biological cell studying and analysis. Nowadays, it uses digital image processing methods for extracting and analyzing the gathered images. Fluorescence microscopy is based on light microscopy and, thus, has an optical resolution limited by the diffraction of light. Targets smaller than Rayleigh's limit, such as cell surface receptors, appear in the image as a bright spot spread over multiple pixels. Quantification problems arise when such small targets are clustered together, as this situation is imaged as a cluster of overlapping bright spots. Despite this limitation, quantification based on conventional fluorescence microscopy is required to understand sub-cellular behaviors [1]. As an example application, part of our research focuses on quantifying the response of immune cells against various pathogens [2]. The current state-of-the-art in image processing in this area revolve around precise location of fluorescent targets [3], [4] by filtering high frequency noise and using Gaussian fitting techniques. The estimated location of the fluorescent targets is sometimes improved by using superresolution techniques [5], [6]. Those methods rely on the sparsity of the fluorescent targets to resolve their location and amount. Their precision comes at the cost of high processing power and extended computational time. Quantitative evaluation methods for fluorescent targets has already been developed by Mutch [7] and Pölönen [8]. Mutch's approach being the deconvolution of clustered targets intensities by modeling the intensity of a single target. Pölönen is directly working on a single cluster, fitting a mixture of diffraction patterns using optimization problem solving techniques.

The goal of this paper is to present two methods that can be used for surface receptors quantification within a given group of cells. A large amount of synthetic microscope images has been analyzed and processed in order to detect relationships between the amount of surface receptors and the processing results. The images have been generated using a simulation tool [9] generating synthetic fluorescent microscope images. The synthetic cells are featuring surface receptors, each stained by a fluorophore. Images are generated by convolving the fluorophore distribution with a model of the bright spot emitted by each of them.

This paper is organized as follow. Section II presents the methodology used to generate synthetic images and presents the approaches taken by AFIM and AFPM to solve the problem. Section III discusses the results obtained from the synthetic images and describes how, from the results, AFIM and AFPM are developed. Finally, Section IV concludes this paper.

\section{Methodology}

We have generated hundreds of synthetic microscope images. Those images feature various amount of cells with various amount of surface receptors. Section II-A details the simulation procedure. Section II-B and Section II-C poses the problem we want to solve by analyzing the average intensity (AFIM) and the amount (AFPM) of the pixels carrying the fluorescent signal respectively.

\section{A. Synthetic images}

A simulation tool [9] was used to generate synthetic images of fluorescently-stained groups cells. The particularity of this tool is to be able to simulate the imaging process undergoing in epi-fluorescence microscopy. It emulates cell populations and the distribution of surface receptors within the cells. Based on the generated distribution of receptors, the tool is simulating the optical behavior of a microscope and the sampling effects of an image sensor.

Three sets of images have been generated, each set is defined by the amount of synthetic cells $N_{c}$ present in the image, either 1,3 or 13 . For each set, synthetic images with a varying amount of surface receptors per cell $N_{r}$ have been generated. In order to gather enough data and have statistically relevant measurements, 100 images have been 

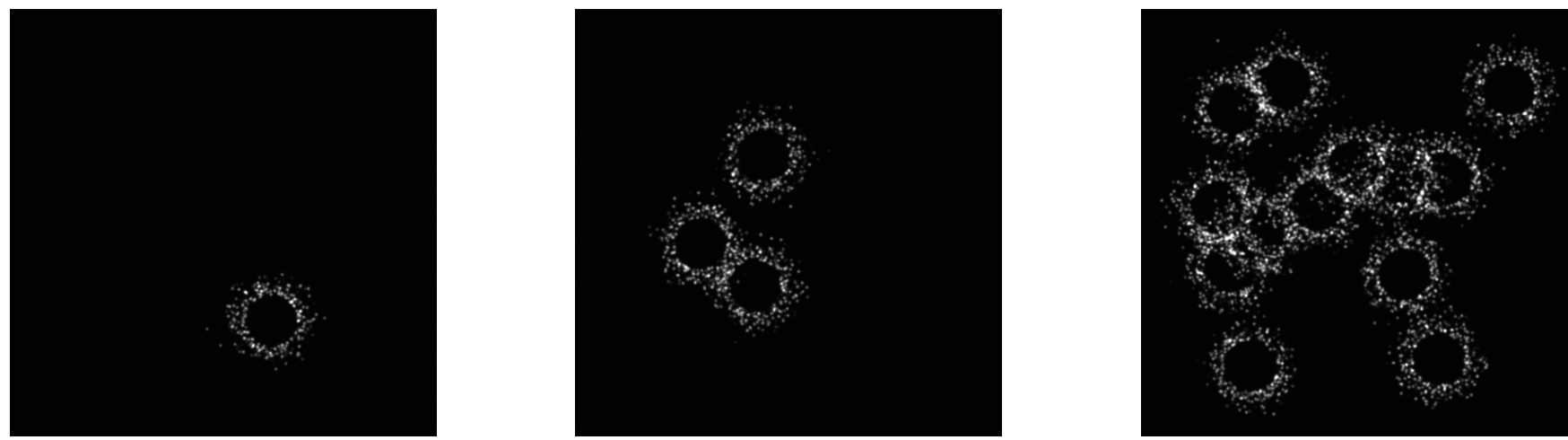

Fig. 1. Examples of generated images prior to segmentation with Ridler's method for 1,3 and 13 cells. Each image covers and area of $52 \mu m$ by $52 \mu m$ on the generated cell samples.

generated for each given $N_{c}$. For example, a configuration defined by $N_{c}=3$ and $N_{r}=1200$ was used to generate 100 images. The location of each receptor is randomized in space and each receptor is assumed to be bound to a single chromophore.

Some examples of synthetic images are found in Fig. 1. The tool was configured in order to generate cells having an approximate diameter of $8 \mu \mathrm{m}$. The simulated microscope an overall magnification of $28 \mathrm{X}$ with a numerical aperture of 0.9 . The simulated imager has a 12 bits resolution and features an array of 500 by 500 square pixels each of size $4.4 \mu \mathrm{m}^{2}$. It has a readout noise $10 \pm 5 e^{-}$and generates dark current at the rate of $10 e^{-} / \mathrm{s}$. The gain was set to $10 e^{-} / \mathrm{ADU}$ (electrons per Analog-to-Digital Units). The simulated exposure time is set to $2 s$ and the light emitted by staining chromophores is $590 \mathrm{~nm}$.

\section{B. AFIM}

The final goal of the research is to detect and measure variations of the cell receptors of an imaged group of cells. The synthetic images have been segmented by Ridler's adaptive thresholding method [10]. This algorithm makes the assumption that target objects, such as the diffraction patterns generated by the chromophores, are having a different average gray level from that of their surrounding. This is why the generated images are having a high enough signal-to-noise ratio, so that their histogram would be bimodal.

For each image and from the computed threshold value $T$, the average fluorescence intensity of all the pixels with an intensity $I>T$ can be computed. This average intensity value is denoted as $\bar{I}_{f}$ and pixels falling under the condition $I>T$ are called fluorescent pixels.

As stated in section II-A, 100 synthetic images were generated for each configuration (i.e. for each couple $\left\{N_{c}, N_{r}\right\}$ ). So, for a given configuration, we assume $\bar{I}_{f}$ to be a random variable out of which we can compute its expected value $E\left[\bar{I}_{f}\right]$ and its standard deviation.

AFIM tries to establish a link between $\bar{I}_{f}$ computed on a fluorescent image and the actual amount of receptors per cell $N_{r}$ for a group of cells. We want to find a function $f(\cdot)$ that embodies the relationship between that measurement and an approximated value $\tilde{N}_{r}$ of the real, unknown, value of $N_{r}$

$$
\bar{I}_{f}=f\left(\tilde{N}_{r}\right)
$$

The results of the processed images are gathered in section III-A where the link $f(\cdot)$ is established by relating the computed expected value $E\left[\bar{I}_{f}\right]$ and the simulation input parameter $N_{r}$.

\section{AFPM}

AFPM follows the same objective as AFIM. The only difference is that we want to find a link $g(\cdot)$ to retrieve an approximated value of the amount of receptors per cell $\tilde{N}_{r}$ from the amount of fluorescent pixels per cell $\bar{N}_{f}$, instead of their average intensity $\bar{I}_{f}$, for a fluorescent image of a given group of cells. Thus the target link can we written as

$$
\bar{N}_{f}=g\left(\tilde{N}_{r}\right)
$$

Similarly, and using the same synthetic images, we assume $\bar{N}_{f}$ to be a random variable and extract its expected value $E\left[\bar{N}_{f}\right]$ and its standard deviation. The results of the processed images are gathered in section III-B where the link $g(\cdot)$ is established by relating the computed expected value $E\left[\bar{N}_{f}\right]$ and the simulation input parameter $N_{r}$.

\section{RESULTS AND DISCUSSION}

Based on the measurements made on the thresholding results, $E\left[\bar{I}_{f}\right]$ and $E\left[\bar{N}_{f}\right]$, we introduce a mathematical formulation to describe $f(\cdot)$ and $g(\cdot)$. Based on the approach taken by Mascetti [11], we link the unknown biological value $N_{r}$ with the measurements made on a fluorescent images using synthetic images.

\section{A. AFIM}

The main idea behind this method is based on the graphs plotted in Fig. 2, showing the evolution of the expected fluorescent pixel average intensity $E\left[\bar{I}_{f}\right]$ versus the amount of receptors per cell $N_{r}$ for three different groups of cells.

Each graph can be divided into two regions. A non-saturated and a saturated region. The former is characterized by fluorescent pixels that are not saturated due to the relative low 


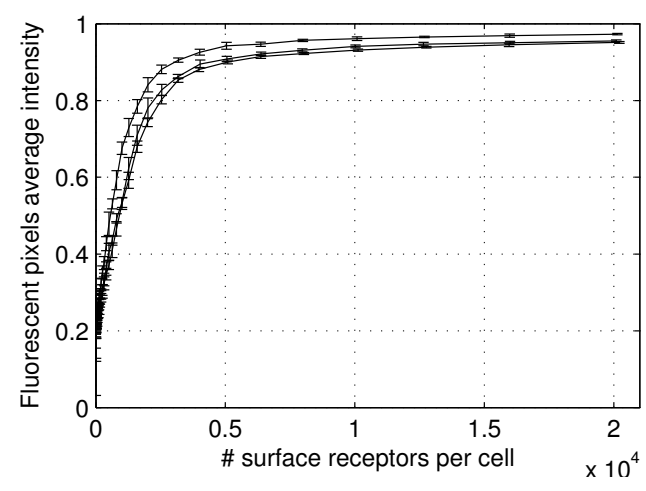

Fig. 2. Normalized average intensity of the fluorescent pixels $E\left[\bar{I}_{f}\right]$ with respect to the amount of receptors per cell $N_{r}$ within the processed images From top to bottom curve, the amount of imaged cells is 13,3 and 1 respectively.

TABLE I

\begin{tabular}{cccc}
\hline Cells & $N_{r}$ & $E\left[\bar{I}_{f}\right]$ & $E\left[\bar{I}_{f}\right]-\beta$ \\
\hline 13 & 20 & $0.231 \pm 0.006$ & $-0.009 \pm 0.006$ \\
13 & 40 & $0.244 \pm 0.007$ & $0.004 \pm 0.007$ \\
\hline 13 & 500 & $0.471 \pm 0.030$ & $0.231 \pm 0.030$ \\
13 & 1000 & $0.676 \pm 0.016$ & $0.436 \pm 0.016$
\end{tabular}

amount of stained receptors in the imaged cells. On the other hand, a high amount of receptors emit too much light and the fluorescent pixels are saturating. This characterizes the saturated region.

AFIM is based on the linear relationship between $E\left[\bar{I}_{f}\right]$ and $N_{r}$ that the non-saturated region exhibits. Relating to (1), we can model $f(\cdot)$ by

$$
\begin{array}{cc} 
& \bar{I}_{f}=f\left(\tilde{N}_{r}\right)=\alpha \tilde{N}_{r}+\beta \\
\Leftrightarrow & \tilde{N}_{r}=\frac{\bar{I}_{f}-\beta}{\alpha}
\end{array}
$$

where $\alpha$ and $\beta$ are constants whose values depend on various experimental parameters. The value of $\beta$ can be estimated by computing the expected value $E\left[\bar{I}_{f}\right]$ for a single receptor (i.e. $N_{r}=1$ ) and assuming $\alpha \ll \beta$. Using a simulation tool like the one presented in [9] and for the experimental setup described in Section II-A, the value of $\beta$ for the graphs in Fig. 2 is estimated as $\beta=0.24$. On the other hand, the value of $\alpha$ cannot be easily estimated without knowing the values of $N_{r}$, which is the unknown in our problem, since it depends on various biological parameters.

Practically, this linear relation allows us to quantitatively evaluate variations in $N_{r}$ as long as we stay in the nonsaturate region. For example, knowing $\beta$, we can easily detect a doubling of $N_{r}$ if the value of $\bar{I}_{f}-\beta$ has doubled. Note this is only true if we are in the non-saturated region but also if the amount of receptors is not too low, as reported in Table I. For very low amount of receptors, the error on measurement $\bar{I}_{f}$ is too high as the light intensity emitted by a receptor depends on its location in space. In focus receptors will appear brighter than out-of-focus ones. In these cases, we can refer to other quantification methods [7], [8].

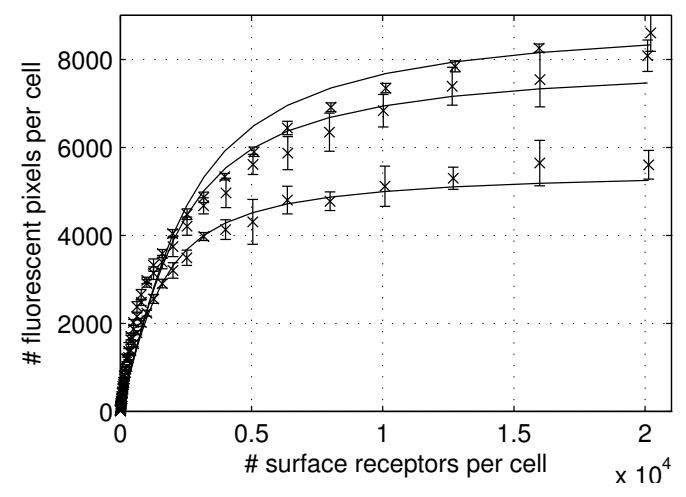

Fig. 3. Number of fluorescent pixels $E\left[\bar{N}_{f}\right]$ with respect to the amount of receptors per cell $N_{r}$ within the processed images. From top to bottom curve, the amount of imaged cells is 1,3 and 13 respectively. $\gamma \delta=2.41$ pixels per receptor and $\gamma \pi / 2=\{5500,8000,9000\}$ pixels respectively.

TABLE II

\begin{tabular}{ccc}
\hline Cells & $N_{r}$ & $E\left[\bar{N}_{f}\right]$ \\
\hline 1 & 400 & $1742 \pm 116$ \\
3 & 400 & $1594 \pm 92$ \\
13 & 400 & $1341 \pm 61$ \\
\hline
\end{tabular}

For estimating $N_{r}$ in the saturated-region, we need to use AFPM, discussed in Section III-B.

\section{B. $A F P M$}

Measurements of the expected amount of fluorescent pixels $E\left[\bar{N}_{f}\right]$ versus the amount of receptors per cell $N_{r}$ are shown in Fig. 3 and in Table II. Fig. 3 displays three graphs, one for each image set (1, 3 or 13 cells). Table II shows quantitative values extracted from the graphs, related to the example images in Fig. 1.

Each group of cell can be described by a fixed curve, similar to the ones presented in Fig. 3. The non-linear characteristic of these curves can be explained by proximity of the receptors or fluorophores. As already introduced, in a fluorescent image, the diffraction pattern of a single chromophore spreads over multiple pixels. Thus, when many chromophores are close enough together (i.e. below Rayleigh's limit), the diffraction patterns will overlap.

As a result, even if a thresholded image of a single infocus fluorophores contains $A$ fluorescent pixels, a thresholded image $N$ clustered chromophores will not contain $N \times A$ fluorescent pixels. Eventually, when there are too many fluorophores, the amount of fluorescent pixels tends to stabilize.

Let's assume we are analyzing a single group of cells. We want to determine the relation $g(\cdot)$ as introduced in (2). The curve describing this relation behaves like an arctangent. When $N_{r}$ is low, the relation is quasi-linear, while for high values of $N_{r}$, the amount of fluorescent pixels is saturating. Thus, we can model $g(\cdot)$ by

$$
\begin{array}{cc} 
& \bar{N}_{f}=g\left(\tilde{N}_{r}\right)=\gamma \arctan \left(\delta . \tilde{N}_{r}\right) \\
\Leftrightarrow & \tilde{N}_{r}=\frac{1}{\delta} \tan \left(\frac{\bar{N}_{f}}{\gamma}\right)
\end{array}
$$


where $\gamma$ and $\delta$ are constants whose values depend on various experimental parameters. Practically, using (6), we can get an approximation on the amount of receptors per cell $\tilde{N}_{r}$ based on the measurement $\bar{N}_{f}$ done on a single fluorescent image. Thus, we need to determine the value of $\gamma$ and $\delta$ bound to the imaged group of cells. A discussion on what $\gamma$ and $\delta$ represent and how to evaluate them is done hereunder.

1) Limit conditions: In order to determine what experimental parameters are influencing the constants $\gamma$ and $\delta$, we need to extract some of the limit conditions of the modeling function (6). First, by computing the following limit

$$
\lim _{\tilde{N}_{r} \rightarrow+\infty} g\left(\tilde{N}_{r}\right)=\frac{\gamma \pi}{2}
$$

we can link the constant $\gamma$ with the size of the group of cells and the distribution of the stained receptors within. This limit extracts the behavior of Ridler's method [10] when the amount of receptors is high. In other words, when an extra receptor in the group of cells do not affect the amount of fluorescent pixels. So, the constant $\gamma \pi / 2$ is the maximum amount of fluorescent pixels that can be detected and $\gamma$ has the unit [pixel].

Second, the parameter $\delta$ can be extracted by analyzing how the amount of detected fluorescent pixels vary for low amount of surface receptors per cell. Mathematically, we have

$$
\frac{d g(0)}{d \tilde{N}_{r}}=\gamma \delta
$$

The resulting expression, $\gamma \delta$, simply defines the average amount of fluorescent pixels that a single receptor will induce in the processed image (i.e average size of the diffraction pattern). The unit for the constant $\gamma \delta$ is [pixels/receptor] and therefore, the unit of $\delta$ is $[1 /$ receptor $]$.

2) Constant evaluation: The first expression we want to evaluate for a given setup is $\gamma \delta$. This can be done using the $\sigma$ parameter of the normalized 2D Gaussian model of the microscope PSF [12]

$$
\sigma=\frac{0.21 \lambda_{e m}}{\mathrm{NA}}
$$

where NA is the numerical aperture and $\lambda_{e m}$ the wavelength of the photons emitted by the chromophores. Approximating the radius of the diffraction pattern of a receptor by $\sigma$, an approximation of the value of $\gamma \delta$ can be

$$
\tilde{\gamma \delta}=\frac{\pi(M . \sigma)^{2}}{p_{a}}
$$

where $M$ is the overall magnification of the optical system and $p_{a}$ is the pixel area on the image sensor.

The evaluation of the constant $\gamma \pi / 2$ can be done after gathering images of the group of cells. Assuming the cells have expressed enough surface receptors, the fluorescent pixel mask (i.e. a binary image) corresponding to a collected image featuring the most fluorescent signal can be used to estimate $\gamma$. A morphological dilation on this mask should be performed using a kernel having the same area, in pixels as $\gamma \delta$. A circular kernel is suited as a chromophore appears on the mask as such.

\section{CONCLUSION AND FUTURE WORK}

Fluorescence detection systems are extensively used nowadays and quantification methods are becoming a major trend. In this paper, we focused on quantification of sub-resolution size targets such as cell surface receptors, taken here as an example. By generating and processing large amount of synthetic fluorescent images, we have extracted two quantification methods for measuring variations and estimating the amount of fluorescently-stained surface receptors. The first method, AFIM, is based on the linear dependency between the fluorescent pixels average intensity with the amount of receptors, assuming a large enough amount of receptors and non-saturated pixels. The second method, AFPM, relies on modeling the amount fluorescent pixels per cell versus the amount of receptors per cell. This method having the advantage of being independent of whether pixels are saturated or not.

\section{ACKNOWLEDGMENT}

This work is supported by the Nutri-CHIP project, which is financed with a grant form the Swiss Nano-Tera.ch initiative and evaluated by the Swiss National Science Foundation. The research was also partially supported by the NanoSys project, within the program ERC-2009-AdG-246810.

\section{REFERENCES}

[1] G. Mascetti, L. Vergani, A. Diaspro, S. Carrara, G. Radicchi, and C. Nicolini, "Effect of fixatives on calf thymocytes chromatin as analyzed by $3 \mathrm{~d}$ high-resolution fluorescence microscopy," Cytometry, vol. 23, no. 2, pp. 110-119, 1996.

[2] G. Vergères, B. Bogicevic, C. Buri, S. Carrara, M. Chollet, L. CorbinoGiunta, L. Egger, D. Gille, K. Kopf-Bolanz, K. Laederach, R. Portmann, Q. Ramadan, J. Ramsden, F. Schwander, P. Silacci, and B. Walther, "The nutrichip project - translating technology into nutritional knowledge,' British Journal of Nutrition, 2012, in press.

[3] E. Peterson and J. Harris, "Quantitative detection of single molecules in fluorescence microscopy images," Analytical chemistry, vol. 82, no. 1, pp. 189-196, 2009.

[4] G. Mashanov and J. Molloy, "Automatic detection of single fluorophores in live cells," Biophysical journal, vol. 92, no. 6, pp. 2199-2211, 2007.

[5] B. Huang, H. Babcock, and X. Zhuang, "Breaking the diffraction barrier: super-resolution imaging of cells," Cell, vol. 143, no. 7, pp. 1047-1058, 2010.

[6] M. Fernández-Suárez and A. Ting, "Fluorescent probes for superresolution imaging in living cells," Nature Reviews Molecular Cell Biology, vol. 9, no. 12, pp. 929-943, 2008.

[7] S. Mutch, B. Fujimoto, C. Kuyper, J. Kuo, S. Bajjalieh, and D. Chiu, "Deconvolving single-molecule intensity distributions for quantitative microscopy measurements," Biophysical journal, vol. 92, no. 8, pp. 2926-2943, 2007.

[8] H. Pölönen, J. Tohka, and U. Ruotsalainen, "Automatic quantification of fluorescence from clustered targets in microscope images," Image Analysis, pp. 667-675, 2009.

[9] J. Ghaye, G. De Micheli, and S. Carrara, "Simulated biological cells for receptor counting in fluorescence imaging," BioNanoSci., vol. 2, no. 2, pp. 94-103, 2012 .

[10] T. Ridler and S. Calvard, "Picture thresholding using an iterative selection method," IEEE transactions on Systems, Man and Cybernetics, vol. 8, no. 8, pp. 630-632, 1978.

[11] G. Mascetti, S. Carrara, and L. Vergani, "Relationship between chromatin compactness and dye uptake for in situ chromatin stained with dapi," Cytometry, vol. 44, no. 2, pp. 113-119, 2001.

[12] B. Zhang, J. Zerubia, and J. Olivo-Marin, "Gaussian approximations of fluorescence microscope point-spread function models," Applied Optics, vol. 46, no. 10, pp. 1819-1829, 2007. 